\title{
Improved Ex-LEACH Protocol based on Energy Efficient Clustering Approach
}

\author{
Pallavi Zambare \\ Department of computer Engineering, \\ Smt.Kashibai Navale College Of Engineering, \\ Off Sinhgad Road.Vadgaon(Bk),Pune-411041, India.
}

\author{
Shital Dabhade \\ Department of computer Engineering \\ Smt.Kashibai Navale College Of Engineering, \\ Off Sinhgad Road.Vadgaon(Bk),Pune-411041, India.
}

\begin{abstract}
Wireless sensor network is a wireless network consisting of autonomous sensors, communicating with each other in dispersed manner to observe the environment. Sensors are commonly fond of to microcontroller and are power-driven by battery. Routing methods are the main issue for networks where resources are restricted. Major challenge of this type of network is to overcome the short life span due to high energy utilization of the batteries in the sensor nodes. So we are providing a routing methodology that improves that network life span and efficiency of wireless sensor network by reducing energy consumption with the proposed cluster based power saving algorithm is called as Ex-LEACH (Extended LEACH).The key idea of this paper is to decrease information transmission distance of sensor nodes in wireless sensor networks by using the homogeneous cluster Method. We calculate the average distance between the sensor nodes and take into consideration the residual energy for selecting the suitable cluster head nodes. So that the energy consumption can be equally distributed between all the sensors node by taking into consideration mainly six factors: Packet delivery ratio, Normalized routing load, End to end packet delay, Throughput, Energy Consumption, Residual Energy. The performance is compared with other similar existing protocols such as LEACH and LEACH-C routing protocols
\end{abstract}

\section{General Terms}

K-Mean Algorithm, Routing algorithm.

\section{Keywords}

Wireless sensor network, clustering, Power saving, LEACH, LEACH-C, Ex-LEACH.

\section{INTRODUCTION}

Wireless Sensor Networks (WSNs) are a special kind of improvised networks. WSN behave like an Ad hoc networks. In WSN routing techniques are the most important subject. It consists of number of small sensor nodes which have limited processing power, memory and sensing ability.

These sensor nodes communicate over short distance via a wireless medium for example, environment monitoring, military surveillance, and industrial process control. If sensor nodes in the sensor network will not able to communicate with the base station directly, then intermediate sensor nodes will used for the data forwarding [3]. Thus, as there intermediate sensor nodes are used for the forwarding the packets to the destination node directly, but this intermediate sensor nodes are consuming the more power of battery and rapidly. To overcome this drawback, a number of routing protocols have been building up for the WSN. The routing protocols for the wired networks cannot be applicable for the WSN.
All the proposed protocols will comes under any of the three types:1) Direct approach 2) Location based routing 3) Attribute based routing.

The easy flooding type routing protocols will be coming below direct approach type. It is not an energy efficient protocol for the sensor networks. In the Location based routing the base station communicates with sensor nodes based on its location uniqueness. Here all the nodes are conscientious of its location through GPS (Global Positioning System) recipients in the network.

In WSN instead of collecting information from all the nodes, the application requires the data only from the nodes which satisfies its concern and this information gathering method is generally called as the data centric approach or attribute based routing. Direct diffusion and rumor routing are the best examples for the attribute based routing or data centric approach.

Other routing methods are cluster based routing and Hierarchical based routing. To organize sensor nodes in network use clustering method. Clustering is method in which sensor nodes in network organize themselves into hierarchical structures. By doing this, sensor node can use the in short provide network resources such as processing power of battery. Inside a cluster, data aggregation and data fusion are achieved. Cluster formation is usually based on left over energy.

LEACH is one of the most excellent hierarchical routing protocols. LEACH (low energy adaptive clustering hierarchy) [5] is a standard version in the hierarchical routing protocol. Leach introduce concept is round. Round consist of two phases' set-up phase and steady phase.

During the setup phase, some device nodes are elected as cluster heads (CHs) in proportion to certain policy and other nodes join in the clusters as member nodes. in the steady-state Phase, the $\mathrm{CHs}$ collect and combined the information coming back from their own cluster members so pass on to a base station (BS).

On the other hand many researchers discovered that LEACH is suffered from some disadvantages. Therefore to beat this, based on leach LEACH-C protocol was introduced which be different from LEACH. In the LEACH-C central algorithm is established for election of $\mathrm{CHs}$ throughout each level of network; though it required every sensor node in the network to transmit their information to distant BS [6].

However, as a result of the LEACH as well as LEACH-C, the more energy consumption caused by the difficult to deal with selecting formula and also the high frequency of re-clustering between detector nodes can cause the uneven energy supply and devastate an explicit quantity of energy within the network. We have to modify the selecting formula and modify the method of selecting $\mathrm{CHs}$.

To avoid such issues, recently the one more approach is presented called Cell-LEACH [8]. In this approach, each cluster is divided into 7 subsections those are called as cells. 
Each cell in the network having cell head which are communicating with the cluster heads directly. They aggregate information of cell and hence they prevent sensors from communicating. This approach had shown better energy utilization as compared to existing LEACH and LEACH-C. However we are here aiming to further investigate the performance of Cell-LEACH against our proposed ExLEACH. In this paper we are presenting the new approach and compare its performance against LEACH and LEACH-C. After that, this paper presents a new improved LEACH algorithm.

The rest of the paper is organized as follows: In section 2 presents the related work, in section 3 discuss the proposed system, Paper is conclude in section 4.

\section{RELATED WORK}

In this section we will present the review over the existing approach called LEACH, LEACH-C along with their pros and cons.

In wireless sensor network LEACH is the most accepted hierarchical cluster based routing protocol. Figure 1.show the topology structure of LEACH. in the communication process, every cluster has a cluster head $(\mathrm{CH})$ which is in charge for creating and controlling a TDMA (time division multiple access) program table used by its member nodes to find when to transmit data packets. In LEACH, the nodes in the organized area are planned into local clusters and the communication procedure is divided into rounds with each round including set-up and steady-state phases

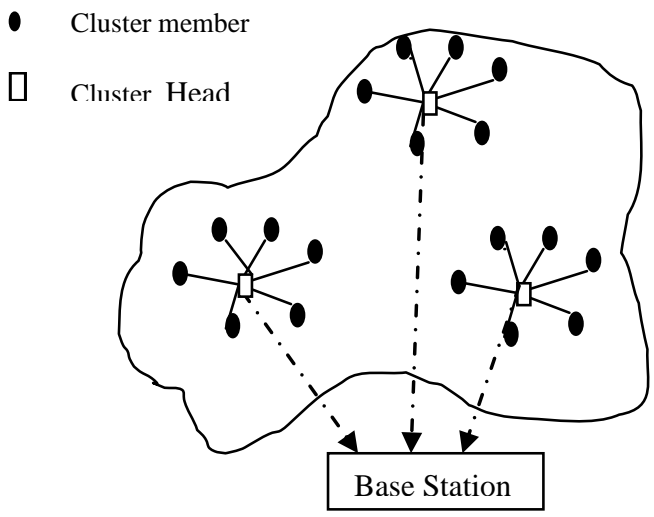

Fig 1: Topology Structure of LEACH

Once some crisis affairs happen within the monitor space, the detector nodes activated to send information to their own cluster head before the remote BS by themselves. The cluster head in the most important collects the info coming back from totally different member nodes and will some aggregation to reduce the redundancy first and so transmits them to BS. in the entire method, the cluster head simply works as a pass on node to help out member nodes abbreviate the transmission distance thus on save energy. As for the set-up and steadystate phases during a round, they will be describes as follows.

\subsection{Set-up Phase}

After final the preparation of detector nodes, every node in the monitor field decides more than a few of alternative nodes whether or not it will become a cluster head within the current round. Throughout the section, every node generates a random range between zero and one and so compares the Threshold value with :

$$
T(n)=\left\{\begin{array}{ll}
\frac{p}{1-p *\left[r \bmod \left(\frac{1}{p}\right)\right]}, & n \in G \\
0 \quad, n \notin G
\end{array} .\right.
$$

Where $\mathrm{p}$ is that the percentage of cluster heads over all nodes within the network, $r$ is that the range of rounds of decision, and $\mathrm{G}$ is that the set of nodes that haven't been selected as cluster heads in round $1 / \mathrm{p}$. The node whose range is larger than the brink can choose itself as a cluster head and so transmits the message to its surround sensing element nodes. Throughout this section, a node may receive over one transmits message from totally different cluster heads, though the node will choose its. Distance to a cluster from the strength of received broadcast signal; the stronger the signal, the nearer to a cluster. Therefore the node whose range is smaller than threshold can only send request message containing its ID to the cluster that has the strongest signal power for saving energy spent on the transmittal distance. Once the cluster head receives request message returning from one node, it records the node's ID and proclaims it as its member node. When the message exchanges between cluster heads and traditional nodes, every $\mathrm{CH}$ gets its own member nodes' data relating to IDs and every fixed node gets that cluster it belongs to. Supported the message it records, the $\mathrm{CH}$ creates a TDMA program table and broadcasts it to the cluster members. Therefore, all the member nodes get their idle slots for information transmission, and so the steady-state section starts.

\subsection{Steady -State Phase}

The organization of a cluster head in every cluster during the set-up phase provides a assurance for the information transmission in a very steady-state phase. In usual situation, member nodes will put off their radio till they sense the necessary surroundings information. If there is some information in have to be obligated to transmit, they'll send the information to $\mathrm{CH}$ throughout the idle slots within the TDMA Program table. As for the CHs, they need to wait communication standing in the least degree times thus on receive the information from totally different member nodes. Once receiving all the information sent by their members, $\mathrm{CHs}$ can combine them first off then send them to BS.

As a result of some sensing element nodes could sense similar surroundings information, the aggregation on the cluster head will reduce inessential information measure worth and communication traffic that features a positive expression to the energy's consumption. Also, the information transmission distance becomes shorter scrutiny with transmission to BS independently for every member node, which may avoid wasting energy for the member nodes. However, the important tasks execution on $\mathrm{CH}$ will result in an extreme amount of energy consumption. So as to avoid creating the $\mathrm{CHs}$ die before time and cause the cascade contact within the network, a replacement round begins and new clusters are restored within the whole network.

\subsection{LEACH-C Protocol}

Based on the LEACH, LEACH-C arranges the sensing element nodes into clusters with every cluster a cluster head and divides a round into set-up and steady state phases. It differs from LEACH exclusively within it uses a high-energy base station to complete the selection of cluster heads.

in the set-up part of every round, each node sends its data with situation to energy to remote BS. Then the BS selects the cluster heads sustained the energy data and broadcasts the IDs of cluster heads to another member node. This method will create the nodes with a lot of energy and a lot of possibility to turn into the cluster head within the present spherical. 
However during this part, each element node must send its ID and energy data to remote BS to compete for the role of cluster heads, which causes energy consumption on the long distance alteration. Equation (2) includes a smart explanation of alteration distance control on the consumption of energy [3]:

$$
E_{\text {trans }}(k, d)=\left\{\begin{array}{ll}
k E_{\text {elec }}+k_{\varepsilon f s} d^{2} & (d<d 0) \\
k E_{\text {elec }}+k_{\varepsilon f s} d^{4} & (d \geq d 0)
\end{array} \cdots\right.
$$

The transmission energy of transmitting a k-bit message over a distance $d$ will cost $E_{\text {trans;efs }} \& E_{m p}$ are the power consumption of transferring one small bit of information in frequent condition. we are able to get that there'll be a specific quantity of energy required to be spent on the transition of energy data for every sensing element node in every round, that cannot be ignored in communication; particularly the BS locates isolated from the monitor field and also the network has a lot of nodes.

\section{Drawbacks of LEACH and LEACH-C:}

Both this protocol which we studied here LEACH and LEACH-C, they conjointly suffer from several drawbacks just like the following.

1. CHs' selection is arbitrary, that doesn't take into consideration the remaining energy of each node or would like the support of BS.

2. The high frequency of re-clustering dissipates a particular amount of energy.

3. It cannot cowl a large space.

4. $\mathrm{CHs}$ aren't equivalently distributed, wherever $\mathrm{CHs}$ will be placed at the sting of the cluster.

\section{PROPOSED SYSTEM}

In this section we will present the algorithms those are under investigation such as Cell-LEACH and proposed Ex-LEACH. In below sections we will give their mathematical models and design.

\subsection{Mathematical Model of Cell-LEACH}

As we studied in ref [1], for this approach sensor network once will be divided in sections which are called cell. Every cell includes many sensors. The sensor node from the cell is chosen as cell head. The cluster is formed by the group of 7 cells those are near to each other. Every cluster has their one cluster head. Clustering and celling will remain the same as long as network is working; just cell-heads and cluster-heads change dynamically.

Below we formulated the two important phases of Cell-

LEACH protocol:

\section{Selection of New Cell Head.}

$\mathrm{I}=\left\{\mathrm{i} \mid \forall\right.$ sensor $_{\mathrm{i}} \in$ cell, Esensori $=\mathrm{E}$ avg-cell $\}$

$\forall \mathrm{i} \in \mathrm{I}$ : if Esensori > Esensori+1

Index $\leftarrow \mathrm{i}$

New Cell-Head $\leftarrow$ sensorIndex.

Where sensori is any sensor in a cell, Esensori is the residual energy of sensor, E avg-cell (as noted in above) is the average energy of cell and i, i+1 and Index are indexes.

\section{Selection of New Cluster Head.}

$\mathrm{J}=\{\mathrm{j} \mid \forall$ sensor $\in$ cluster, Esensor $\mathrm{E}=\mathrm{E}$ avg-cluster $\}$

$\forall \mathrm{j} \in \mathrm{J}$ : if Esensorj > Esensorj+1

Index $\leftarrow \mathrm{j}$

New Cluster-Head $\leftarrow$ sensorIndex

Where sensorj is nothing but the any sensor node in cluster, Esensorj is sensor node residual energy, Eavg-cluster is cluster average energy and j, j+1 and Index are indexes.

\subsection{Mathematical Model of Ex-LEACH}

Thus further we have to present new extended approach to get better the power efficiency as well as extending the lifetime of sensor nodes for the wireless sensor networks. We presented the efficient control saving method which is based on the centralized clustering approach. This new protocol is named as Ex-LEACH which is having aim of extending the lifetime of WSN. In Ex-LEACH we are considering that BS receives location information as well as left over energy for each sensor node and the average remaining energy can be calculated.

If the sensor node remaining energy is higher as compared to average remaining energy, then that sensor node is consider as the cluster head candidate. The algorithm used here is based on k-means algorithm in order to make an ideal allocation for sensor node clusters by using the information of location as well as remaining energy for all sensor nodes. There are two major steps involved for this proposed work such as set-up and steady-state phases. Following is the mathematical model for both phases:

\subsubsection{Set-up Phase}

Basic aim of this step is to make clusters as well as locate cluster head nodes. During the set-up phase, the BS collects the information of the location and energy level from all sensor nodes in the networks. Based on the distinctiveness of stationary sensor nodes, the suitable initial means of points for clusters can be obtained. Let $\mathrm{C}$ be the center location for all sensor nodes. If there are $\mathrm{n}$ sensor nodes in the wireless sensor networks, $\mathrm{C}$ can be calculated by:

$$
C=\frac{\sum_{i=1}^{n} X_{i}}{n} \cdots(3)
$$

Where, $\mathrm{Xi}$ is the coordinate of sensor node $\mathrm{i}$.

Let $\mathrm{R}$ be the average distance between $\mathrm{C}$ and all sensor nodes, which can be calculated by:

$$
R=\frac{\sum_{i=1}^{n}\left|X_{i}-C\right|}{n} \cdots
$$

According to $\mathrm{C}$ and $\mathrm{R}$, the locations of initial mean of point mi (mix , miy $)$ for the cluster $\mathrm{i}$ is calculated by:

$$
\begin{aligned}
& m_{i x}=R * \cos \left(\frac{360}{k} *(i-1) * \frac{\pi}{180}\right)+C_{x} \cdots(5) \\
& m_{i y}=R * \sin \left(\frac{360}{k} *(i-1) * \frac{\pi}{180}\right)+C_{y} \cdots(6)
\end{aligned}
$$

where $\mathrm{k}$ is the number of clusters and $\mathrm{i}=1,2, \ldots, \mathrm{k}$. The first point setting is very important in WSN. It can reduce the iteration time for creating clusters appreciably. As we studied in [2], there are many things have done for setting the initial points. The final clusters are formed when each sensor node is unchanging in the cluster. the flowchart of the initial cluster processing for Ex-LEACH.

The initial cluster processing for our proposed scheme. The cluster head is a sensor node which is closer to the final mean of point and the residual energy of the sensor node is higher than the average residual energy in each cluster. Finally, the cluster architecture is created. The BS broadcasts the routing information of the clusters to all sensor nodes. Hence, each sensor node has its own routing table and knows its task (e.g., cluster head or non-cluster head). Also, each sensor node knows the distances from any other sensor node in its cluster and thereby calculates the transmission power.

After all above process, depending on the total number of sensor nodes within the cluster, the cluster head node creates a program based on Time Division Multiple Access (TDMA) to Allocate the time for the cluster members. 


\subsubsection{Steady-state phase}

Once the clusters are created and therefore the TDMA program is fixed firmly, data transmission will begin. The non-cluster head nodes send data to cluster head node throughout their chosen TRM. Once all the information is received, the cluster head node performs signal process to compress the information into one signal. Then, this signal is shipped to the BS. The quantity of data is reduced thanks to the information aggregation done at the cluster head node. This round is finished and therefore the next round begins with set-up and steady-state phases repeatedly. To avoid unnecessary nodes control messages transmission and control overhead of the BS, the clusters are re-created only when the sensor node cannot work in a convinced round. So, the scheming overhead is only cluster head electing in the most set-up phase.

\section{CONCLUSION}

Finally in the conclusion, one of challenge of WSNs is power saving. Therefore every time researchers needs to work over power saving of WSN and hence enhancing the lifetime of same. Network overhead and sensor nodes load are influencing the overall system lifetime significantly. To enhance the system lifetime performance, distributing the load over sensor nodes. Thus in this project, we conducted our studies over the such distributed protocol called as hierarchical protocol which is having the main functionality of reducing the overall system path loading mechanism by distributing the energy consumptions among the sensor nodes. In this paper we discussed and presented new approaches of energy efficiency based on LEACH hierarchical routing protocol for WSN. We presented pros and cons of LEACH and LEACH-C. Further presented the working of CellLEACH and proposed Ex-LEACH protocol along with their mathematical models. For future work we will suggest to work over the evaluation study of this both protocols and compare their performances in terms of Energy consumption and Throughput.

\section{REFERANCE}

[1] "An Improvement on LEACH Protocol (Cell-LEACH)", Arezoo Yektaparast*, Fatemeh-Hoda Nabavi**, Adel Sarmast*, *Department of Computer Engineering, Science and Research Branch, Islamic Azad University, Khouzestan, Iran.

[2] "An efficient cluster-based power saving scheme for wireless sensor networks", Jau-YangChang* and PeiHao Ju, Chang and Ju EURASIP Journal on Wireless Communications andNetworking 2012, 2012:172 http://jwcn.eurasipjournals.com/content/2012/1/172

[3] N. Israr, I. Awan, “ Multihop ClusteringAlgorithm for Load Balancing In Wireless SensorNetworks," I. J. of simulation vol. 8 , no. 1 .

[4] E. Abdellah, S. Benalla, A. Benihssane, and M. Hasnaoui, "Advanced Low Energy AdaptiveClustering Hierarchy," (IJCSE) International Journal on Computer Science and Engineering, vol.02, no. 07, pp. 2491-2497, 2010.
[5] W.R.Heinzelman, A. Chandrakasan, and H. Balakrishnan, "Energy-efficient communication protocol for wireless microsensor networks," in Proceedings of the 33rd Annual Hawaii International Conference on System Siences (HICSS '00), January 2000.

[6] W. B. Heinzelman, A. P. Chandrakasan, and H. Balakrishnan, "An application-specificprotocol architecture for wireless microsensor networks," IEEE Transactions on WirelessCommunications, vol. 1, no. 4, pp. 660-670, 2002.

[7] J. Xu, G. Yang, Z.-Yu. Chen, L. Chen, and Z. Yang, "Performance analysis of data aggregation algorithms in wireless sensor networks," in Proceedings of the InternationalConference on Electrical and Control Engineering (ICECE '11), September 2011.

[8] Q Xuegong, C Yan, A control algorithm based on double cluster-head for heterogeneouswireless sensor network, in Proc Industrial and Information Systems (IIS), pp. 541-544 (July2010)

[9] Y-U Yun, J-K Choi, N Hao, S-J Yoo, Location-based spiral clustering for transmission scheduling in wireless sensor networks, in Proc Advanced Communication Technology (ICACT), pp. 470-475 (February 2010)

[10] HD Tarigh, M Sabaei, A new clustering method to prolong the lifetime of WSN, in Proc Computer Research and Development (ICCRD), pp. 143-148 (March 2011).

[11] H. Abusaimeh and S. H. Yang, "Dynamic cluster head for lifetime efficiency in WSN,"International Journal of Automation and Computing, vol. 6, no. 1, pp. 48-54, 2009.

[12] V.Chandrasekaran, Dr.A.Shanmugam,"A REVIEW ON HIERARCHICAL CLUSTER BASED ROUTING IN WIRELESS SENSOR NETWORKS"Volume 3, No.2, February 2012 Journal of Global Research in Computer Science

[13] Mr. Halke Rajesh, Mrs. Kulkarni V. A.Design of Enhanced LEACH Routing Protocol for Wireless Sensor Network,IOSR Journal of Electronics and Communication Engineering (IOSR-JECE) ISSN: 22782834, ISBN: 2278-8735, PP: 07-12

[14] Lin SHEN and Xiangquan SHI, "A Location Based Clustering Algorithm for Wireless Sensor Networks"international journal of intelligent control and systems vol. 13, no. 3, september 2008,208-213

[15] Fuzhe Zhao, You Xu, and Ru Li”Improved LEACH Routing Communication Protocol for a WirelessSensorNetwork" Hindawi Publishing Corporation International Journal of Distributed Sensor Networks Volume 2012, Article ID 649609, doi:10.1155/2012/649609

[16] Shio Kumar Singh, M P Singh and D K Singh."Routing Protocols in Wireless Sensor Networks - A Survey" International Journal of Computer Science and Engineering Survey (IJCSES) Vol.1, No.2, November 2010. 